\title{
(6) OPEN ACCESS \\ Interprofessional collaboration among care professionals in obstetrical care: are perceptions aligned?
}

\author{
Anita Romijn, ${ }^{1}$ Pim W Teunissen, ${ }^{2}$ Martine $C$ de Bruijne, ${ }^{1}$ \\ Cordula Wagner, ${ }^{1,3}$ Christianne J M de Groot ${ }^{2}$
}

${ }^{1}$ Department of Public and Occupational Health, Amsterdam Public Health research institute, VU University Medical Center, Amsterdam, The Netherlands

${ }^{2}$ Department of Obstetrics and Gynaecology, VU University Medical Center, Amsterdam, The Netherlands

${ }^{3}$ Netherlands Institute for Health Services Research, Utrecht, The Netherlands

\section{Correspondence to} Anita Romijn, Department of Public and Occupational Health, Amsterdam Public Health research institute, VU University Medical Center, 1081 BT Amsterdam, Netherlands; a.romijn@vumc.nl

Received 13 December 2016 Revised 28 June 2017 Accepted 21 July 2017 Published Online First 26 September 2017
(D) Check for updates

To cite: Romijn A, Teunissen PW, de Bruijne MC, et al. BMJ Qual Saf 2018;27:279-286.

\begin{abstract}
Background In an obstetrical team, obstetricians, midwives and nurses work together in a dynamic and complex care setting. Different professional cultures can be a barrier for effective interprofessional collaboration. Although the different professional cultures in obstetrical care are well known, little is understood about discrepancies in mutual perceptions of collaboration. Similar perceptions of collaboration are important to ensure patient safety. We aimed to understand how different care professionals in an obstetrical team assess interprofessional collaboration in order to gain insight into the extent to which their perceptions are aligned. Methods This cross-sectional study was performed in the north-western region of the Netherlands. Care professionals from five hospitals and surrounding primary-care midwifery practices were surveyed. The respondents consisted of four groups of care professionals: obstetricians ( $n=74)$, hospital-based midwives known as clinical midwives $(n=42)$, nurses $(n=154)$ and primary-care midwives $(n=109)$. The overall response rate was $80.8 \%$. We used the Interprofessional Collaboration Measurement Scale (IPCMS) to assess perceived interprofessional collaboration. The IPCMS distinguishes three subscales: communication, accommodation and isolation. Data were analysed using non-parametrical tests.

Results Overall, ratings of interprofessional collaboration were good. Obstetricians rated their collaboration with clinical midwives, nurses and primarycare midwives more positively than these three groups rated the collaboration with obstetricians. Discrepancies in mutual perceptions were most apparent in the isolation subscale, which is about sharing opinions, discussing new practices and respecting each other. Conclusion We found relevant discrepancies in mutual perceptions of collaboration in obstetrical care in the Netherlands. Obstetrical care is currently being reorganised to enable more integrated care, which will have consequences for interprofessional collaboration. The findings of this study indicate opportunities for improvement especially in terms of perceived isolation.
\end{abstract}

\section{INTRODUCTION}

In obstetrics, interprofessional collaboration is essential for patient safety. Poor collaboration and communication failure are known causes of adverse events. ${ }^{12}$ The Joint Commission on Accreditation of Healthcare Organisations identified breakdown in communication as the leading cause $(72 \%)$ of perinatal death or disability in a root analysis of 47 cases. $^{3}$ In most cases, communication failure was attributed to organisation culture, for example, hierarchy and failure to function as a team. In an obstetrical team, obstetricians, midwives and nurses need to work together in a dynamic and complex care setting. These care professionals are required to be skilled top players in their own discipline and they must also be effective team players. ${ }^{45}$

Differences between professional cultures can be an important barrier for interprofessional collaboration. ${ }^{6}$ Obstetricians and midwives are known to have different views on pregnancy and childbirth as a result of differences in education, responsibilities and patient populations. ${ }^{7}$ Midwives take care of women with low-risk pregnancies who require minimal medical intervention. Obstetricians take charge when complications arise during pregnancy or childbirth. ${ }^{7-10}$ Differences between professional cultures can negatively affect open communication, mutual trust, working towards shared goals and a clear understanding of each team member's tasks and responsibilities. ${ }^{6} 11$ These are important aspects of effective collaboration and if they are not in place, that can make interprofessional collaboration difficult and increase the risk of patient harm.

In multidisciplinary teams it is important that ideas about patient care needs and perceptions of collaboration among care 
professionals are aligned to ensure patient safety. ${ }^{1}{ }^{12}$ Previous studies in various healthcare settings have focused on discrepancies in perceptions towards teamwork and interprofessional collaboration. ${ }^{12-16}$ These studies have predominantly looked into differences between nurses and physicians. A common pattern is that physicians have a more positive perception of their collaboration with nurses compared with nurses' perceptions. ${ }^{12} 13$ Different views of patient needs, difficulties in speaking up and the lack of input from nurses in decision-making, or poor reception of that input, are some explanations cited for the discrepancies. $^{12-14} 17$

Although the different professional cultures in obstetrical care are well known, little is understood about perceptions among all team members of collaboration. Besides studies on nurse-physician collaboration, perceived interprofessional collaboration within an obstetrical team including midwives has not been examined yet. Insight into possible discrepancies is important in order to improve collaborative practice and thus enhance patient safety. We aimed to understand how different care professionals in obstetrical teams assess their collaboration in order to gain insight into the extent to which their perceptions are aligned. Therefore the research question for this study was: What discrepancies are there in the perceptions of interprofessional collaboration in multidisciplinary teams consisting of obstetricians, nurses and midwives?

\section{METHODS}

\section{Context}

We performed a cross-sectional study in the northwestern region of the Netherlands, as part of the larger LOCoMOTive study (Local Obstetrical Collaboration Multidisciplinary Onsite Team training effectiveness $s t u d y)$. The LOCoMOTive study is a multicentre study aimed at increasing understanding of the implementation and impact of team training and tools for structured communication such as SBARR (Situation, Background, Assessment, Recommendation, Read back). The details of the LOCoMOTive study are described elsewhere. ${ }^{18}$ We included care professionals working in multidisciplinary teams from five Local Obstetrical Collaborations (LOCs). A LOC refers to all obstetrical care professionals working in a specific regional area: an obstetrics department in a hospital plus the surrounding primary-care midwifery practices.

In the Netherlands, independent primary-care midwives take care of women in the locality with low risks of pathology. Women are referred to a hospital if risks of adverse fetal or maternal outcomes are high, or if complications arise during pregnancy or childbirth. In the hospital, obstetricians take over responsibility and care, in collaboration with nurses and hospital-based midwives, also called clinical midwives. ${ }^{9}{ }^{10}$ A consequence of this system is a high referral rate between care settings for women, in which close collaboration is required.
Box 1 Interprofessional Collaboration

Measurement Scale (IPCMS)

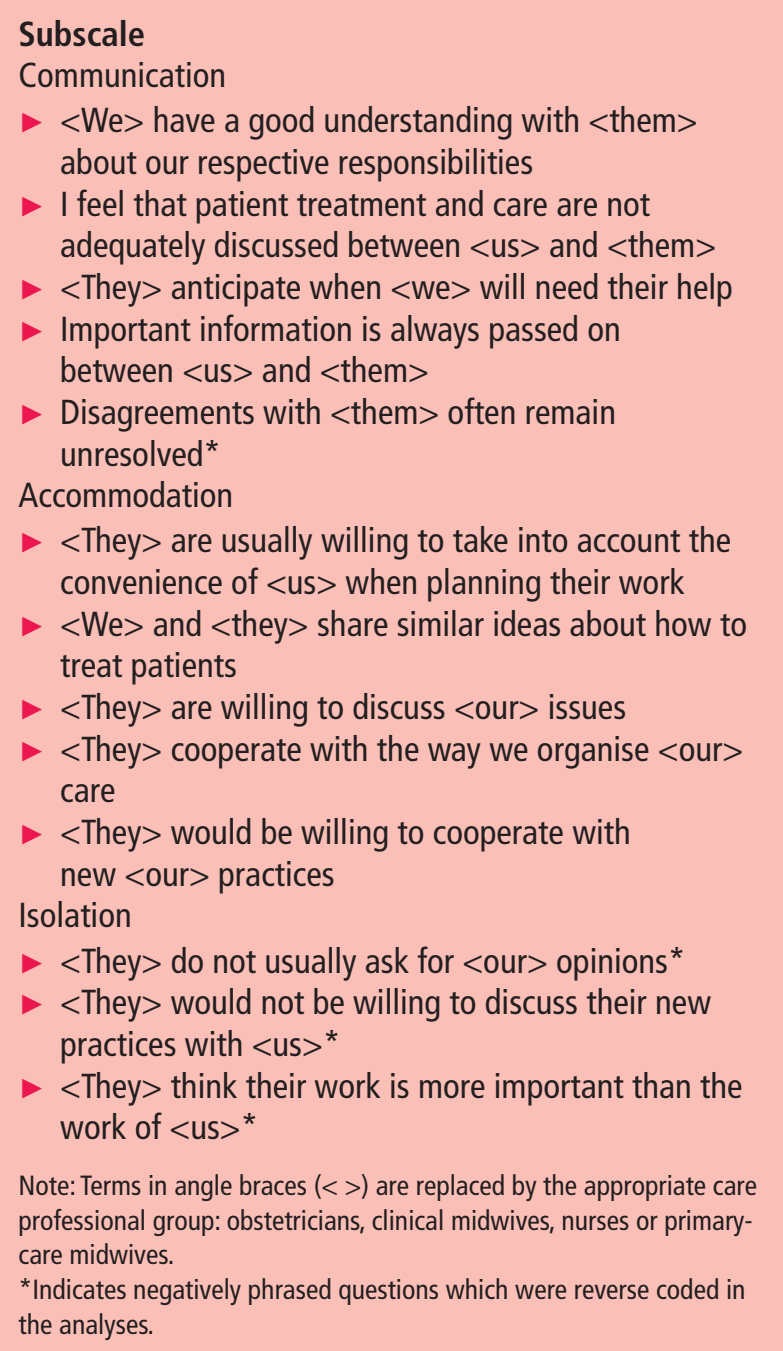

\section{Measurement}

To assess perceptions of interprofessional collaboration, all care professionals in the study were invited to complete the Interprofessional Collaboration Measurement Scale (IPCMS). The IPCMS is a 13-item questionnaire which is suitable for multiple care professional groups. ${ }^{19}$ The questionnaire distinguishes three subscales related to interprofessional collaboration, namely 'communication', 'accommodation' and 'isolation'. The 'communication' and 'accommodation' subscales consist of five questions each. The 'isolation' subscale consists of three questions. The questions are rated on a 4-point Likert Scale $(1=$ strongly disagree to $4=$ strongly agree). The questionnaire was translated into Dutch using consensus meetings with three translators. Box 1 shows the questions and related subscales of the IPCMS.

The IPCMS has a round robin design, which enables all care professional groups to act as raters as well as subjects in the questionnaire. ${ }^{19}$ In this study, obstetricians (including registrars in obstetrics and gynaecology), clinical midwives, nurses 
Table 1 Characteristics of the respondents

\begin{tabular}{lcccc}
\hline & Obstetricians & Clinical midwives & Nurses & $\begin{array}{c}\text { Primary-care } \\
\text { midwives }\end{array}$ \\
\hline Total (N) & 74 & 42 & 154 & 109 \\
$N(\%)$ & $59(79.7)$ & $40(95.2)$ & $152(98.7)$ & $107(98.2)$ \\
$\quad$ Female & & & & \\
Median (Q1-Q3) & $38(32-47)$ & $47(37-52)$ & $45(34-54)$ & $35(30-44)$ \\
$\quad$ Age in years & $40(36-46)$ & $29(24-36)$ & $28(24-32)$ & $40(33-48)$ \\
$\quad$ Working hours per week & $4(3-9)$ & $10(5-17)$ & $15(7-26)$ & $10(5-19)$ \\
$\quad$ Work experience in profession in years & $2(1-10)$ & $4(2-9)$ & $12(5-20)$ & $8(4-13)$ \\
\hline
\end{tabular}

and primary-care midwives were treated as four different care professional groups. In accordance with the round robin design, all care professional groups filled in the 13 -item questionnaire three times (once for each of the other three groups) in order to enable interprofessional comparisons between all professions. The mutual assessments between all possible pairs of professions resulted in 12 assessments of interprofessional collaboration (obstetricians rating collaboration with clinical midwives, nurses and primary-care midwives; clinical midwives rating collaboration with obstetricians, nurses and primary-care midwives; nurses rating collaboration with obstetricians, clinical midwives and primary-care midwives, and primary-care midwives rating collaboration with obstetricians, clinical midwives and nurses).

\section{Data collection}

Data collection took place between October 2013 and October 2014. All care professionals in the five participating LOCs were invited to fill in the questionnaire. Every obstetrician (or registrar), clinical midwife, nurse and primary-care midwife received a personal link to an online survey via email. Every participant was given 2 months to complete the questionnaire. During this period, two reminders were sent to those who had not yet completed the questionnaire. The survey included questions on respondent characteristics and room for comments in addition to the IPCMS questionnaire.

\section{Data analyses}

Descriptive statistics were used to study the respondent characteristics of sex, age, working hours and working experience. In order to answer the research question, we investigated discrepancies in perceptions of interprofessional collaboration in terms of communication, accommodation and isolation. The IPCMS questions were assigned to the subscales of communication, accommodation and isolation (box 1). Cronbach's $\alpha$ s were calculated for the IPCMS subscales to test for internal consistency. The data for the subscales were skewed, clustered towards response options 3 and 4. These response options reflect generally good collaboration ratings. Therefore, the data were analysed using non-parametrical tests.

To assess possible differences in the perceptions of interprofessional collaboration, we used the MannWhitney $\mathrm{U}$ test to test for differences between the care professional groups. For example, the communication score for 'obstetricians rating primary-care midwives' was compared with the communication score for 'primary-care midwives rating obstetricians'. This test was repeated for every interprofessional comparison on subscale level as well as on item level. A significance level of $p \leq 0.05$ was considered to indicate statistical significance. Analyses were performed using SPSS V.20.

\section{RESULTS}

In total, 395 of out 469 care professionals completed the online questionnaire. We excluded 16 incomplete questionnaires $(4.1 \%)$, resulting in a total of 379 questionnaires for analyses. Completed questionnaires were returned by obstetricians $(n=74$, response rate $88.1 \%)$, clinical midwives $(n=42$, response rate 95.1\%), nurses $(\mathrm{n}=154$, response rate $71.0 \%)$ and primary-care midwives $(n=109$, response rate $87.9 \%)$. The overall response rate was $80.8 \%$, taking all obstetrical care professionals in the five LOCs into account. Table 1 shows the respondents' characteristics. Because of skewed data, the median and lower and upper quartiles are shown.

Table 2 shows the results of the interprofessional comparisons between obstetricians, clinical midwives, nurses and primary-care midwives for the subscales 'communication', 'accommodation' and 'isolation'. Possible within-group differences between five LOCs were considered. However, no substantial differences were found. The significant differences $(p<0.01)$ between care professionals are visualised in figure 1 . Below, the results are discussed on subscale level as well as on item level.

\section{Communication}

Cronbach's $\alpha$ for the communication subscale ranged between 0.47 and 0.68 . These $\alpha$ s fall below the generally accepted lower limit of $0.70 .^{20}$ Thus, 
Table 2 Results of interprofessional comparisons for the subscales 'communication', 'accommodation' and 'isolation'

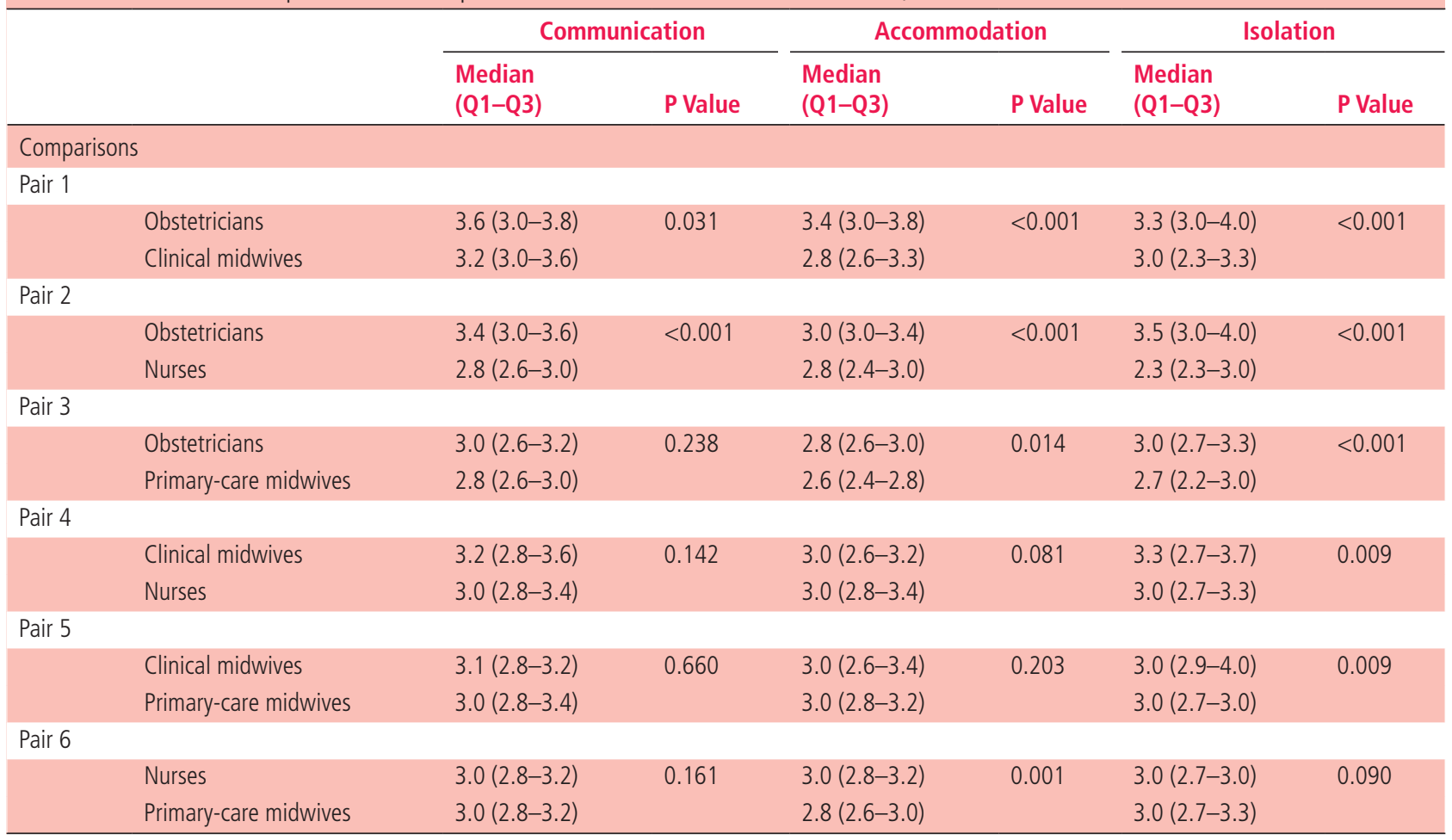

results from this subscale should be interpreted with caution. As shown in table 2, significant differences for the subscale communication were found between obstetricians and nurses $(p<0.001)$ as well as between obstetricians and clinical midwives $(p=0.031)$. Obstetricians reported a high score for communication with

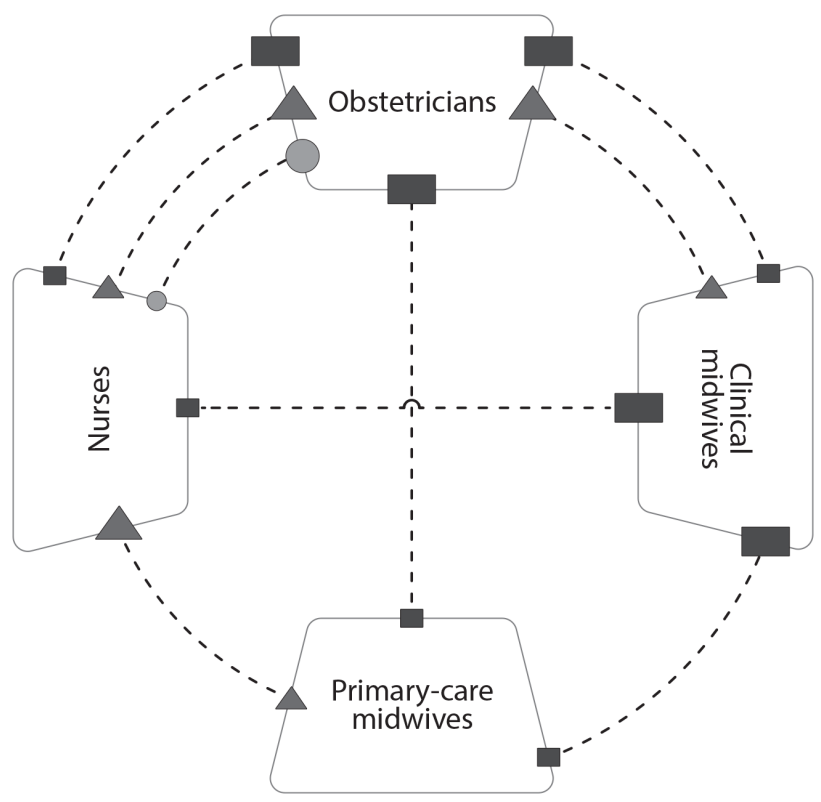

Figure 1 Discrepancies in mutual perceptions of 'communication', 'accommodation', 'isolation' in obstetrical care. 'Circle': Communication subscale; 'Triangle': Accommodation subscale; 'Square': Isolation subscale. The professions with a large circle, triangle or square reported a significantly higher score compared with the professions with a small symbol $(p \leq 0.01)$. clinical midwives $(3.6,3.0-3.8)$ and with nurses (3.4, $3.0-3.6)$, but received a significantly lower score from clinical midwives $(3.2,3.0-3.6)$ as well as from nurses $(2.8,2.6-3.0)$. We found no significant differences in other interprofessional comparisons for the subscale communication.

The communication subscale consists of five items, as shown in box 1, and we tested for item-level differences between care professionals. In four out of five items, obstetricians rated a significantly higher score compared with nurses. The item 'Important information is always passed on between $<u s>$ and $<$ them $>$ ' was the only exception. None of the interprofessional comparisons showed a significant difference for this item. The item ' $\langle$ We $\rangle$ have a good understanding with <them> about our respective responsibilities' showed significant lower scores of nurses compared with obstetricians $(\mathrm{p}<0.001)$, clinical midwives $(\mathrm{p}=0.009)$ and primary-care midwives $(\mathrm{p}=0.017)$. The item ' $<$ They $>$ anticipate when $<$ we $>$ will need their help' showed significant differences between all interprofessional comparisons, with the exception of the comparison between clinical midwives and primary-care midwives.

\section{Accommodation}

Cronbach's $\alpha$ for the accommodation subscale ranged between 0.75 and 0.82 . Significant differences for the subscale accommodation were found between obstetricians and clinical midwives $(p<0.001)$, between obstetricians and nurses $(p<0.001)$ as well as between obstetricians and primary-care midwives $(\mathrm{p}=0.014)$. 
Obstetricians reported a high score for accommodation with clinical midwives $(3.4,3.0-3.8)$, nurses (3.0, 3.0-3.4) and primary-care midwives $(2.8,2.6-3.0)$. However, obstetricians received a significant lower score for accommodation from these three groups of care professionals. In addition, a significant difference was found between nurses and primary-care midwives $(p=0.001)$. Nurses reported a high score for accommodation with primary-care midwives $(3.0,2.8-3.2)$, but received a significant lower score from this care professional group $(2.8,2.6-3.0)$.

The accommodation subscale consists of five items and we tested for item-level differences between care professionals. The interprofessional comparisons showed a significantly higher score of obstetricians compared with nurses on all items. In four out of five items, obstetricians rated a significantly higher score compared with clinical midwives. The item ' $<$ They $>$ are willing to discuss <our $>$ issues' was the only exception. In addition, this item was rated higher by nurses compared with primary-care midwives. Moreover, the following two items were rated higher by nurses compared with primary-care midwives as well as clinical midwives; ' $<$ We $>$ and $<$ they $>$ share similar ideas about how to treat patient' and ' $<$ They $>$ cooperate with the way we organise <our $>$ care'.

\section{Isolation}

Cronbach's $\alpha$ for the isolation subscale ranged between 0.62 and 0.72 . All interprofessional comparisons for the subscale isolation showed discrepancies in mutual perceptions, except for collaboration between nurses and primary-care midwives. Significant differences were found between obstetricians and clinical midwives $(p<0.001)$, between obstetricians and nurses $(p<0.001)$ as well as between obstetricians and primary-care midwives $(\mathrm{p}<0.001)$. The negatively phrased questions were reverse coded in the analyses and, therefore, a higher score indicates a more positive perception. Obstetricians reported a high score for isolation with clinical midwives $(3.3,3.0-4.0)$, nurses $(3.5,3.0-4.0)$ and primary-care midwives (3.0, 2.7-3.3). However, obstetricians received a significantly lower score for isolation from these three groups of care professionals. Also, significant differences were found between clinical midwives and nurses $(p=0.009)$ and between clinical midwives and primary-care midwives $(p=0.009)$. Clinical midwives reported a high score for isolation with nurses (3.3, 2.7-3.7) and primary-care midwives (3.0, 2.9-4.0), but received a significantly lower score from these two groups of care professionals (respectively, 3.0, 2.7-3.3 and 3.0, 2.7-3.0).

The isolation subscale consists of three items and we tested for item-level differences between care professionals. The interprofessional comparisons showed a significantly higher score of obstetricians compared with clinical midwives, nurses and primary-care midwives on all items. The item ' $<$ They $>$ would not be willing to discuss their new practices with <us>' showed the only significant difference between obstetricians and registrars in this study. Registrars rated a higher score on this item compared with obstetricians. In addition, clinical midwives rated the item ' $<$ They $>$ do not usually ask for <our> opinions' significantly higher compared with primary-care midwives. The item ' $<$ They $>$ think their work is more important than the work of <us >' showed significant differences for all interprofessional comparisons.

\section{DISCUSSION}

This study showed multiple discrepancies in mutual perceptions of interprofessional collaboration between obstetricians, clinical midwives, nurses and primary-care midwives. Overall, ratings of interprofessional collaboration were good. However, obstetricians rated their collaboration with clinical midwives, nurses and primary-care midwives more positively than these three groups rated the collaboration with obstetricians. The interprofessional collaboration between clinical midwives, nurses and primary-care midwives showed fewer significant discrepancies. Discrepancies in mutual perceptions were most apparent in the isolation subscale, which is about sharing opinions, discussing new practices and respecting each other.

The findings are in line with previous research on interprofessional collaboration and teamwork in other healthcare settings. A more positive perception of collaboration by physicians compared with nurses and other team members was also reported in, for example, the intensive care setting and in the operating theatre. ${ }^{12131516}$ For example, Wauben et $a l^{12}$ studied discrepant perceptions of collaboration and related concepts in surgical teams. They found differences between surgeons and other team members concerning teamwork, communication and situational awareness. ${ }^{12}$ Thomas et $a l^{13}$ studied perceptions of teamwork in intensive care units. In this study too, physicians reported the most favourable perceptions of collaboration. ${ }^{13}$ The current study showed that this pattern is also applicable to perceptions of collaboration among obstetrical care professionals in the Netherlands and more specifically to different subscales of collaboration; communication, accommodation and isolation.

There are several ways to explain the misaligned perceptions between care professionals in obstetrical care in the Netherlands. First, the organisation of obstetrical care in the Netherlands leads to a physical distance between primary-care midwives in the local community and obstetricians, clinical midwives and nurses in hospitals. This is cited as an important barrier to interprofessional collaboration and facilitates the existence of different professional cultures. ${ }^{21}$ Second, the care professional groups collaborate in different ways and frequencies. Primary-care midwives take care 
of women with low risks of pathology. When women are referred during labour due to complications, primary-care midwives may choose to stay or not with labouring women. In hospitals, clinical midwives manage the majority of births in collaboration with nurses. ${ }^{22}$ Obstetricians are responsible for these women and are involved when additional risks or problems occur. These differences in status and responsibilities are also an important third aspect to explain the misaligned perceptions. ${ }^{621}$ Finally, the organisation of obstetrical care is subject of debate in the Netherlands. Uncertainty about changing roles and responsibilities may affect perceptions of collaboration. ${ }^{23}$

The isolation subscale showed most discrepancies between all care professionals. The items of this subscale cover the following topics: sharing opinions, willingness to discuss new practices and respecting each other. In addition, the other subscales showed most discrepancies for the items; understanding responsibilities, anticipating when help is needed (communication) and sharing similar ideas (accommodation). All these items relate to the importance of cohesion and team identity. For successful interprofessional collaboration it is necessary to expand care professionals' social identity beyond their profession. A common team-based identity, rather than a profession-based identity, encourages team members to perceive themselves as part of one superordinate group. $^{24} 25$ In addition, cohesion refers to the force that drives team members to stay together and want to work together. ${ }^{26}{ }^{27}$ Team members who feel no sense of cohesion, or feel isolated, are less motivated and less likely to collaborate to meet the team goals. ${ }^{27}$ In particular, it is important to address cohesion and team identity to improve collaborative practice.

Efforts to improve collaborative practice often include team training interventions. Different training programmes have been developed, such as Crew Resource Management (CRM) and Team Strategies and Tools to Enhance Performance and Patient Safety (TeamSTEPPS). ${ }^{28}$ These interventions focus on comparable teamwork competencies, such as communication, situational awareness, leadership, role clarity and coordination. Findings indicate that CRM and TeamSTEPPS training produces positive reactions from trainees as well as improving attitudes towards collaboration. ${ }^{28-31}$ These team training interventions could help to increase shared understanding and reduce discrepancies in perceptions of collaboration.

\section{Methodological considerations}

We used IPCMS for its round robin design to gain insight into contrasting views of interprofessional collaboration. IPCMS includes important aspects of effective teamwork, although the subscales are not as comprehensively defined in order to compare the content with the concept of teamwork. IPCMS was originally designed to measure interprofessional collaboration for multiple health provider groups, physicians, nurses and allied health professionals. The latter group encompasses a wide range of professionals from different training backgrounds that cannot be accurately represented by one care provider group. ${ }^{19}$ The current study population involved well defined groups of professionals. Therefore, we decided to include four care professional groups as raters and subjects in the survey. Due to the multiple comparisons, there is a higher chance of false-positive results, type 1 error.

The internal consistency of the subscales was calculated with Cronbach's $\alpha$. In the original IPCMS paper by Kenaszchuk et al, ${ }^{19}$ the consistency was estimated with Raykov's composite reliability statistic. ${ }^{19}$ The Raykov values ranged between 0.73 and 0.82 (communication), between 0.79 and 0.88 (accommodation), between 0.71 and 0.79 (isolation). The estimates of reliability produced by composite reliability are, on average, larger than those estimated by Cronbach's $\alpha .^{32}$ In this current study, the communication subscale showed poor internal consistency. ${ }^{20}$ This indicates that more research is needed on the translation and validity of the IPCMS questionnaire in different healthcare settings. Still, the subscales of accommodation and isolation demonstrated reasonable internal consistency in our study.

A limitation of this study is the assessment of care professionals as homogenous groups, which is inherent to the research question and questionnaire set-up. Respondents were able to elaborate on their answers to the questionnaire in the comment fields. However, some care professionals used this space to comment on the questionnaire itself. The most common comments seemed to be related to the perceived subjective nature and difficulty in generalising. For example, a respondent stated "We work with so many different people that it differs from person to person. I don't think I can give a right answer in general". We realise that collaboration can differ between individuals within groups of care professionals. There are always some care professionals who are less willing to collaborate and communicate than others.

The strengths of this study are the round robin comparisons, the high response rate limiting selection bias and the relevance of this study's topic for Dutch obstetrical care. Referrals between primary-care midwives and obstetricians are an important point for attention in improving collaboration and the continuity of care. ${ }^{33}$ Obstetrical care in the Netherlands is now reorganising towards integrated care and enhancing the continuity of care from the perspective of the patient. This change has consequences for collaboration between all care professionals within an LOC, for example, by creating joint electronic record systems and shared protocols and decision-making. ${ }^{34}$ Therefore, it would be interesting to repeat this survey over time to monitor changes in perceptions of interprofessional collaboration. 
In conclusion, care professionals in obstetrical care do not always have similar perceptions of communication, accommodation and isolation. This study provided insight into the perception of interprofessional collaboration by all members of teams of obstetrical care professionals. Though the effect of discrepant perceptions on patient safety was not studied, the findings increase awareness that obstetricians generally rate collaboration more positively compared with clinical midwives, nurses and primary-care midwives. Especially discrepant perceptions for the isolation subscale indicate a potential for improving collaborative practice.

Acknowledgements We would like to thank the care professionals who participated in this study.

Contributors All authors made contributions to the design of the study. AR carried out data collection, performed statistical analyses, interpreted results and drafted the manuscript. PWT, $\mathrm{MCdB}, \mathrm{CW}$ and CJMdG made contributions to interpretation of the data and revisions to the manuscript. All authors read and approved the final manuscript.

Funding This study was supported by ZonMw, the Dutch Organisation for Health Research and Development - grant number 209020001.

Competing interests None declared.

Ethics approval Medical Ethical Committee of the VU University Medical Center (Amsterdam, The Netherlands).

Provenance and peer review Not commissioned; externally peer reviewed.

Open Access This is an Open Access article distributed in accordance with the Creative Commons Attribution Non Commercial (CC BY-NC 4.0) license, which permits others to distribute, remix, adapt, build upon this work noncommercially, and license their derivative works on different terms, provided the original work is properly cited and the use is non-commercial. See: http://creativecommons.org/licenses/ by-nc/4.0/

(C) Article author(s) (or their employer(s) unless otherwise stated in the text of the article) 2018. All rights reserved. No commercial use is permitted unless otherwise expressly granted.

\section{REFERENCES}

1 Leonard M, Graham S, Bonacum D. The human factor: the critical importance of effective teamwork and communication in providing safe care. Qual Saf Health Care 2004;13(suppl 1):i85-i90.

2 Manser T. Teamwork and patient safety in dynamic domains of healthcare: a review of the literature. Acta Anaesthesiol Scand 2009;53:143-51.

3 Joint Commission on Accreditation of Healthcare Organizations. Preventing infant death and injury during delivery. The Joint Commission. Sentinel Event Alert Issue 30, 2004.

4 Fitzgerald A, Davison G. Innovative health care delivery teams: learning to be a team player is as important as learning other specialised skills. J Health Organ Manag 2008;22:129-46.

5 McCallin A. Interdisciplinary practice-a matter of teamwork: an integrated literature review. J Clin Nurs 2001; 10:41928.

6 Hall P. Interprofessional teamwork: professional cultures as barriers. J Interprof Care 2005;19(suppl 1):188-96.
7 Reime B, Klein MC, Kelly A, et al. Do maternity care provider groups have different attitudes towards birth? BJOG 2004;111:1388-93.

8 Ratti J, Ross S, Stephanson K, et al. Playing nice: improving the professional climate between physicians and midwives in the Calgary area. J Obstet Gynaecol Can 2014;36:590-7.

9 Amelink-Verburg MP, Buitendijk SE. Pregnancy and labour in the Dutch maternity care system: what is normal? the role division between midwives and obstetricians. J Midwifery Womens Health 2010;55:216-25.

10 De Vries R, Nieuwenhuijze M, Buitendijk SE. What does it take to have a strong and independent profession of midwifery? Lessons from the Netherlands. Midwifery 2013;29:1122-8.

11 D'Amour D, Ferrada-Videla M, San Martin Rodriguez L, et al. The conceptual basis for interprofessional collaboration: core concepts and theoretical frameworks. J Interprof Care 2005;19(Suppl 1):116-31.

12 Wauben LSGL, Dekker-van Doorn CM, van Wijngaarden JDH, et al. Discrepant perceptions of communication, teamwork and situation awareness among surgical team members. Int J Qual Health Care 2011;23:159-66.

13 Thomas EJ, Sexton JB, Helmreich RL. Discrepant attitudes about teamwork among critical care nurses and physicians. Crit Care Med 2003;31:956-9.

14 Lyndon A, Zlatnik MG, Wachter RM. Effective physiciannurse communication: a patient safety essential for labor and delivery. Am J Obstet Gynecol 2011;205:91-6.

15 Miller PA. Nurse-physician collaboration in an intensive care unit. Am J Crit Care 2001;10:341-50.

16 Nelson GA, King ML, Brodine S. Nurse-physician collaboration on medical-surgical units. Medsurg Nurs 2008;17:35-40.

17 Zwarenstein M, Reeves S. Working together but apart: barriers and routes to nurse-physician collaboration. Jt Comm J Qual Improv 2002;28:242-7.

18 Romijn A, de Bruijne MC, Teunissen PW, et al. Complex social intervention for multidisciplinary teams to improve patient referrals in obstetrical care: protocol for a stepped wedge study design. BMJ Open 2016;6:e011443.

19 Kenaszchuk C, Reeves S, Nicholas D, et al. Validity and reliability of a multiple-group measurement scale for interprofessional collaboration. BMC Health Serv Res 2010;10:83.

20 Tavakol M, Dennick R. Making sense of Cronbach's alpha. Int J Med Educ 2011;2:53-5.

21 Weller J, Boyd M, Cumin D. Teams, tribes and patient safety: overcoming barriers to effective teamwork in healthcare. Postgrad Med J 2014;90:149-54.

22 Cronie D, Rijnders M, Buitendijk S. Diversity in the scope and practice of hospital-based midwives in the Netherlands. $J$ Midwifery Womens Health 2012;57:469-75.

23 Ministry Health Welfare and Sports. Drie rapporten zwangerschap en geboorte en reactie op zorgstandaard integrale geboortezorg (Reports on pregancy and chilbirth and response to integrated care). The Hague: Ministry Health Welfare and Sport, 2016. Document: 958229-149541-CZ 2016.

24 Bartunek JM. Intergroup relationships and quality improvement in healthcare. BMJ Qual Saf 2011;20(suppl 1):i62-i66.

25 Mitchell RJ, Parker V, Giles M. When do interprofessional teams succeed? investigating the moderating roles of team and 


\section{Original research}

professional identity in interprofessional effectiveness. Human Relations 2011;64:1321-43.

26 Michan S, Rodger S. Characteristics of effective teams: a literature review. Aust Health Rev 2000;23:201.

27 Salas E, Grossman R, Hughes AM, et al. Measuring Team Cohesion: observations from the Science. Hum Factors J Hum Factors Ergon Soc 2015;57:365-74.

28 Weaver SJ, Dy SM, Rosen MA. Team-training in healthcare: a narrative synthesis of the literature. BMJ Qual Saf 2014;23:359-72.

29 Salas E, Wilson KA, Burke CS, et al. Does crew resource management training work? an update, an extension, and some critical needs. Hum Factors 2006;48:392-412.

30 Buljac-Samardzic M, Dekker-van Doorn CM, van Wijngaarden JDH, et al. Interventions to improve team effectiveness: A systematic review. Health Policy 2010;94:183-95.
31 Rabøl LI, Østergaard D, Mogensen T. Outcomes of classroombased team training interventions for multiprofessional hospital staff. A systematic review. Qual Saf Health Care 2010;19:e27.

32 Peterson RA, Kim Y. On the relationship between coefficient alpha and composite reliability. J Appl Psychol 2013;98:194-8.

33 Stuurgroep zwangerschap en geboorte. Een goed begin: Veilige zorg rond zwangerschap en geboorte (A good start: safe care during pregnancy and childbirth). Stuurgroep zwangerschap en geboorte. Utrecht, 2009.

34 Perdok H, Jans S, Verhoeven C, et al. Opinions of maternity care professionals and other stakeholders about integration of maternity care: a qualitative study in the Netherlands. $B M C$ Pregnancy Childbirth 2016;16:188. 